\title{
Elementos para el diseño de un parque científico-tecnológico en la Facultad de Ingeniería Industrial de la UNMSM
}

Orestes Cachay Boza* Adolfo Acevedo Borrego** Carolina Linares Barrantes $* * *$

\section{RESUMEN}

Este artículo muestra los resultados del estudio Proyecto del parque tecnológico de la Facultad de Ingeniería Industrial. Se refiere a elaborar los elementos que permitan desarrollar un estudio de factibilidad para el Parque Científico Tecnológico también el alcance de las áreas temáticas, el modelo de gestión y organización, y las características competitivas. Los logros se plantean a dos niveles. A nivel de diseño se ha formulado el proyecto de un parque considerando su definición, a partir de su visión, misión y objetivos, características competitivas y modelo de gestión y organización, su posible evolución y visión de futuro para llegar a fases superiores.

Se concluye, principalmente, que el diseño del proyecto de Parque Científico-Tecnológico, el estudio de factibilidad y su posterior implantación, ha de representar un ámbito dedicado a la producción de conocimiento y creación de bienes tecnológicos, incubadora de tecnologías y productos de avanzada, centro de oportunidades de desarrollo y negocio.

La importancia para la sociedad está en función de la capacidad del parque para irradiar sus logros: población y comunidad académica, la posibilidad de desarrollar sus propios proyectos de investigación y generar actividades de innovación y productivas que contribuyan a generar conocimiento nacional y desarrollar tecnología que genere capacidad competitiva, local y global.

Palabras clave: parque científico-tecnológico, elementos competitivos, producción de conocimiento

\section{ELEMENTS FOR DESIGN OF SCIENTIFIC AND}

TECHNOLOGICAL PARK AT INDUSTRIAL ENGINEERING FACULTY OF UNMSM

\section{ABSTRACT}

This paper shows achievement of study Project Scientific and Technological Park at Industrial Engineer Faculty. Its relates to elaborate the elements in order to develop a feasibility study for the Scientific and Technological Park, and the scope of the subject areas, the model of management and organization, and competitive features. The achievements raises two levels. At design level is formulated a draft park considering its definition, from its vision, mission and objectives, competitive features and management model and organization of PCT, possible evolution and future vision to reach higher phases.

The main conclusion is that design of the proposed Science and Technology Park Project, the feasibility study and subsequent implementation, must represent an area dedicated to the production of knowledge and creation of technological goods incubator advanced technologies and products, center of business and development opportunities.

The importance fos society is based on the ability of the park to radiate its achievement: public and academic community, the opportunity to develop their own research projects and generate innovation and productive activities that contribute to generating knowledge and developing technology to generate competitive products, local and global.

Keywords: science and technology park, competitive elements, knowledge production

\section{INTRODUCCIÓN}

La función central del ingeniero industrial es crear, mejorar y optimizar los procesos industriales [8], que complementa con actividades de investigación aplicada e innovación, a fin de cubrir los fines empresariales, de satisfacer necesidades a través de las tareas de diseñar nuevos productos y servicios, mejorar la calidad de productos y servicios y reducir costos de operación, empleando el conocimiento y la tecnología moderna para obtener cambios en procesos industriales y en estructuras físicas y conceptuales [1].

Para cumplir el nuevo rol en la innovación y emprendimiento, se debe considerar la posibilidad de diseñar e implantar un espacio geográfico de influencia, denominado Parque Científico-Tecnológico (PCT), el que ha de representar un ámbito dedicado a la producción de conocimiento y creación de bienes tecnológicos, incubadora de tecnologías y productos de avanzada, centro de intercambio de oportunidades de desarrollo y negocio, irradiando a la posibilidad de desarrollar proyectos de investigación.

El objetivo del presente estudio es mostrar los resultados del estudio Proyecto del parque tecnológico de la Facultad de Ingeniería Industrial de la UNMSM, se refiere a elaborar los elementos que permitan desarrollar un futuro estudio de factibilidad para un proyecto de Parque Científico-Tecnológico, definiendo el alcance de las áreas temáticas del PCT, el modelo de gestión y organización y las características competitivas para plantear convenios con empresas e instituciones.

\section{ANTECEDENTES DE LOS PARQUES CIENTÍFICO-TEC- NOLÓGICOS}

Un parque científico-tecnológico es un modelo de gestión de conocimiento para el desarrollo. Es un ambiente físico que permite la integración empresa-universidad-gobierno [10], que facilita el acceso, gestión y empleo del conocimiento científico y tecnológico, el intercambio y libre flujo de ideas, la generación y el compartimiento de experiencia académica y de negocio entre

\footnotetext{
Profesor en la Facultad de Ingeniería Industrial, Departamento de Producción y Gestión Industrial de la UNMSM. E-mail: orestescachay@yahoo.es

** Profesor en la Facultad de Ingeniería Industrial, Departamento de Producción y Gestión Industria de la UNMSM. E-mail: aacevedo@speedy.com.pe

*** Profesor en la UPG de la Facultad de Ingeniería de Sistemas e Informática de la UNMSM E-mail: klinares@speedy.com.pe
} 
las comunidades universitaria y empresarial [5]. Siempre se les ha considerado como lugares para el desarrollo de oportunidades empresariales [9].

\subsection{Antecedentes históricos}

A fines del siglo XIX e inicios del siglo XX, como parte del negocio inmobiliario, surgen en Estados Unidos, los primeros parques industriales, definidos como centros geográficos para realizar actividades de negocio. El concepto inicial era proporcionar un área urbanizada donde se ubicarían empresas productivas.

Su desarrollo es sostenido y creciente en el mundo entero, a partir de la segunda guerra mundial. Se inician como centros de compra y venta, son ambientes de negocios, ingreso a un sector y establecimiento de contratos y acuerdos empresariales, derivan en centros productivos y de generación de empleo, se amplía a entornos de desarrollo e incubadora de nuevos negocios y tecnologías, luego buscan integrar ciudad-universidad-parque como centro neurálgico para investigación y solución de problemas de crecimiento económico, producción de bienes y servicios tecnológicos y creación de fuentes de trabajo [14].

\subsection{Antecedentes académicos}

Los grandes empresarios de USA percibieron el potencial competitivo de la ubicación de sus industrias en entornos geográficos cercanos a las universidades, ya que permitiría el aprovechamiento de la labor educativa y de investigación académica para un mejor posicionamiento en los mercados [4].

Los gobiernos de diversos países consideran que la integración universidad-parque tecnológico representa un valioso medio para el desarrollo económico social, cuyas sinergias redundarían en innovación, crecimiento productivo y estabilidad social [3]. Países europeos y asiáticos, como España, Japón, Tailandia, Singapur, China, Colombia han planteado programas de desarrollo en innovación e investigación para la producción, basados en parques científicos-tecnológicos [24]. Paradójicamente, en dirección contraria a las modas económicas de subsidiariedad del rol productivo del Estado.

\subsection{Parque científico-tecnológico y Universidad}

Los parques industriales integrados a las universidades devienen en los parques científicos-tecnológicos. A nivel de gobiernos se les considera agentes primordiales en el sistema de investigación e innovación del país [21], su consolidación en una región eleva el atractivo para inversiones y propicia la creación e implantación de líneas de investigación científica y programas del desarrollo tecnológico.

Las razones para la creación de un PCT integrado a la Universidad son las siguientes:

- La implantación de proyectos PCT, significa una herramienta clave para el desarrollo y un estímulo para el crecimiento de negocios.

- El PCT impulsa el desarrollo económico de las regiones y localidades donde se ubican.

- Incentiva la expansión de inversiones y promueve el surgimiento de iniciativas emprendedoras en una localidad.

- Revitaliza la dinámica económica en las empresas de la zona.

- Eleva las probabilidades de éxito de las empresas que se ubiquen en el parque industrial.

\subsection{PCT en el Perú}

A partir de indagaciones preliminares, se ha observado que diversas universidades publicitan el inicio de la implantación de su propio parque científico-tecnológico, aunque este se encuentran a nivel de propuestas generales y convenios básicos sin plazos definidos ni proyectos concretos para la implantación [11]. Existen dos organizaciones privadas que, por razones publicitarias, se irrogan el nombre de parque tecnológico, la primera es una pequeña empresa dedicada a brindar seminarios focalizados, la otra es una consultora en software, pero ninguna posee la capacidad ni los objetivos de un parque.

El principal intento de creación de un primer parque tecnológico en el país, se encuentra en Arequipa, con el proyecto Mistisoft cuya puesta en marcha se prevé para el año 2015 o posterior, este PCT estaría orientado, principalmente, a software informático.

Comparativamente, en diversos países de elevado y mediano desarrollo, como España, India, Brasil [23], Chile, se han desarrollado parques tecnológicos gestionados conjuntamente por entes privados y gobiernos, con diferente grado de énfasis en su gestión y decisión.

\section{EL MARCO TEÓRICO DE PCT}

\subsection{Definición de parque científico-tecnológico}

PCT se define como un eficaz instrumento para la transferencia de tecnología, creación y atracción de empresas con elevado valor agregado [13]. Un PCT 
tiene la finalidad de cobijar empresas en su etapa inicial de lanzamiento y consolidación, brindando los medios para el desarrollo de tecnología y fomento de innovación, con la coordinación de gobiernos locales y regionales, organismos privados, asociaciones civiles, empresa y universidad [17].

También se define como una organización que vende o alquila terrenos y edificios a empresas y organizaciones con actividades de investigación y desarrollo de nuevos productos o procesos. Es un espacio de calidad en infraestructura y servicios de información y comunicación que aporta valor y ventaja competitiva en mercados globales [18].

\subsection{Tipos de PCT}

Las diferentes formas que han adquirido los parques, dependiendo de las condiciones espaciales e históricas específicas del contexto geográfico en que se han desarrollado [16], han permitido la definición de cuatro tipos de PCT:

a) Complejos industriales de alta tecnología, como Sillicon Valley en USA.

b) Ciudades científicas, como la ciudad de ciencia multinuclear de Kansai en Japón.

c) Parque tecnológico para atraer empresas de alta tecnología, como Hsinchu en Taiwán.

d) Tecnópolis, como instrumentos de desarrollo regional y descentralización industrial como la Cartuja en Sevilla, España [8].

\subsection{Los factores clave de PCT}

Los factores determinantes para el éxito y el desempeño de un PCT son estos:

- Localización geográfica con potencial de crecimiento.

- Definición de áreas de investigación científica.

- Fomento de incubadoras de pequeños emprendimientos.

- Elevado grado de integración con el entorno (urbano o rural), universidad y empresa.

- Formulación de planes de desarrollo de PCT.

- Programa de innovación y competitividad.

- Programa de proyectos in situ de la comunidad.

- Programa de emprendimiento.

- Programa la mujer profesional en investigación.

- Programa de calidad de vida.

\subsection{La visión de un PCT en la Facultad de Ingeniería Industrial de la UNMSM}

Desde la UNMSM, considerada la primera universidad del país, se debe llegar hacia la comunidad empresarial, a través de las líneas de investigación [20], redefinidas en las facultades de empresa, de manera que difunda y se perciba el potencial de la capacidad científico-técnica de la UNMSM, a través de la oferta de $\mathrm{I}+\mathrm{D}+\mathrm{i}+\mathrm{e}$, según lo requiere la dinámica de crecimiento económico de la reciente década pasada. Se han de identificar las necesidades de investigación y tecnológicas de los mayores sectores productivos, para el desarrollo, aplicación y comercialización de las tareas $1+D+i+e$ generados en la UNMSM.

En concordancia con la visión, misión y plan estratégico de investigación [22], el Vicerrectorado de Investigación ha de consolidar a la UNMSM en el ambiente empresarial mediante contratos entre universidad y empresa. Mediante una Oficina de Transferencia de Investigación se buscaría que los resultados de investigaciones realizadas en la UNMSM, trasciendan el ámbito del laboratorio.

Entre sus actividades se consideraría la promoción de cursos con el objetivo de transferencia de tecnología, donde diversos grupos interdisciplinarios desarrollen sus investigaciones, dando lugar a un número dado de spin-off y patentes. Spin-off se define como empresa nueva formada por miembros del centro de investigación de la universidad, con la finalidad de transferir conocimiento y brindar a los investigadores la posibilidad de llevar a la práctica los resultados de sus proyectos.

\section{ELEMENTOS PARA EL DISEÑO DE UN PCT}

\subsection{Definición y objetivo de un parque científico y tecnológico}

Parque científico y tecnológico es una organización gestionada por profesionales especializados, cuyo objetivo fundamental es incrementar la riqueza de su comunidad promoviendo la cultura de la innovación y la competitividad de las empresas e instituciones instaladas en el parque o asociadas a él [12].

Desde una perspectiva emprendedora, un parque tecnológico estimula y gestiona el conocimiento y tecnología entre universidades, centros de investigación, empresas y mercados [15], impulsando la creación y el crecimiento de empresas innovadoras mediante mecanismos de incubación, incluyendo productos y servicios de valor añadido, dentro de un espacio físico centralizado o distribuido, 
Figura 1. Efectos de un PCT

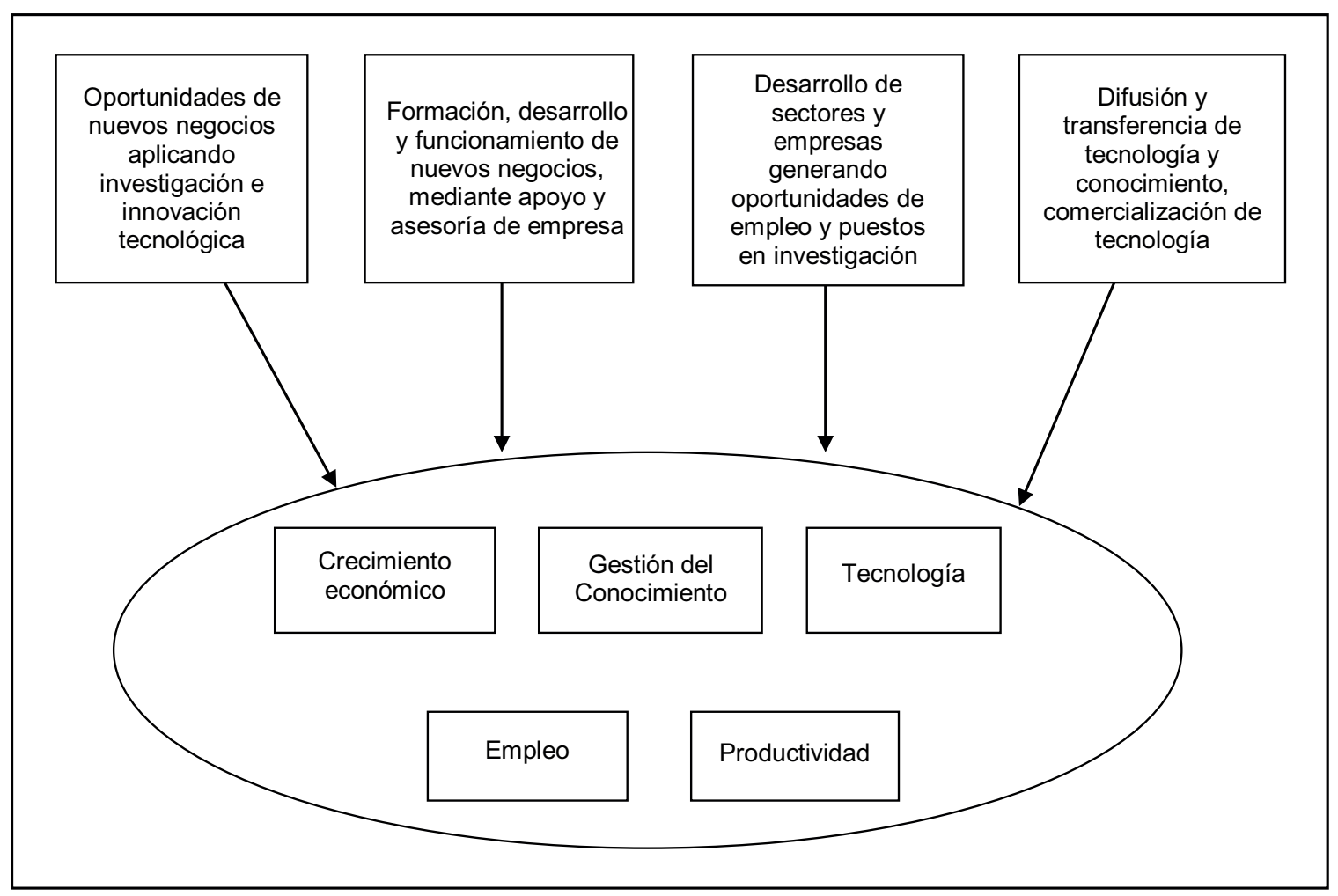

Fuente: Elaboración propia.

conteniendo instalaciones que permiten el desarrollo de tareas de innovación, la transferencia de tecnología, la creación de negocios.

La Figura 1 presenta los beneficios esperados de un PCT.

\subsection{Los elementos de competitividad de un PCT}

Los elementos competitivos de un PCT, se resumen a continuación:

- Ubicación geográfica. Por su efecto en costos, el PCT se ubica cercano las fuentes de recursos o cercano a los mercados de consumo de conocimiento, incluyendo cercanía a vías de comunicación, como puertos, aeropuertos, carreteras.

- Infraestructura de apoyo a investigación. Comprende los servicios básicos de energía eléctrica, sanitarios, comunicaciones, seguridad, administración.

- Administración. En los parques tecnológicos existen profesionistas especializados en la gestión y administración de servicios y en atender necesidades de las empresas que residen en ellos.
- Apoyo del gobierno. Es frecuente que los parques tecnológicos reciban apoyo del gobierno, mediante la creación de un fondo, programas especiales o fideicomisos.

\subsection{El modelo de gestión y organización de un PCT}

\section{Gestión y organización del PCT}

Se plantean cinco modelos de organización de gestión de PCT, estos son:

- Gestión por una universidad, como el caso de parques en México.

- Gestión por el gobierno central.

- Gestión por un ente autónomo con funciones especializadas.

- Gestión por promotores privados sin intervención estatal ni universidad.

- Gestión por organizaciones no gubernamentales con fines sociales.

\section{Las funciones básicas del PCT}

Un PCT desarrolla cuatro tipos de funciones básicas: 
- Adopción y transferencia de tecnología. A partir de la adopción de la innovación, se realiza la difusión y la transferencia de tecnología a nivel sectorial-empresarial y a nivel geográfico, para su adaptación y explotación.

- Desarrollo de innovaciones. Mejorando la tecnología tradicional y generando tecnología de avanzada.

- Creación de factores competitivos. Que permita un entorno académico, científico y de investigación donde se ubiquen geográficamente los factores de competitividad.

- Incubadora de negocios. Para la creación, lanzamiento y marcha normal de empresas basadas en tecnología.

\section{Funciones de apoyo del PCT}

Las funciones generales para el funcionamiento del PCT serían las siguientes:

- Gestionar espacios para el desarrollo de actividades de centros de investigación o empresas de servicios, planta manufacturera, emprendimiento, innovación tecnológica, inicio e incubación de nuevos negocios.

- Brindar servicios de información y consultoría para establecimiento de organizaciones de negocio, desde las alianzas, el financiamiento, tutoría, diseño de proyectos y planes de negocio, capacitación d tipo formal.

- Brindar servicios de apoyo y servicios básicos para el funcionamiento de la actividad de investigación e innovación, incluyendo gestión de proyectos, estudio de mercado, transferencia de tecnología.

- Fomento de nuevos negocios, actividades de marketing y la promoción, para el desarrollo empresarial.

\section{Áreas temáticas de un PCT}

Un parque científico tecnológico debe enfocarse en las siguientes áreas:

- Tecnologías de avanzada en informática, software.

- Tecnologías en sectores tradicionales y de alta rentabilidad como minería y pesquería.

- Tecnologías para gestión y manejo del medio ambiente y reservas ecológicas.

- Tecnologías en industrias de servicios.

- Tecnologías en sectores emergentes y de alto potencial de creación de valor y generación de empleo productivo, como agroindustria, textil, turismo y artesanía.

\section{CAPACIDADES DISTINTIVAS PARA GENE- RAR COMPETITIVIDAD}

\subsection{Capacidad de crear sinergias}

Según la red mundial de parques científicos y tecnológicos [12], los PCT promueven el desarrollo económico y la competitividad de las regiones y ciudades mediante:

- Creación de nuevas oportunidades de negocio y añadir valor para madurar las empresas.

- Fomento del espíritu empresarial y la incubación de nuevas empresas innovadoras.

- Generación de empleos basada en conocimientos.

- Construcción de espacios atractivos para los trabajadores del conocimiento.

- Creación y mejora de sinergia entre universidades y empresas.

\subsection{Resultados entregables que debe brindar un PCT}

EI PCT representa un servicio universitario que está compuesto por distintas áreas que responden a las necesidades de creación y transferencia de tecnología, innovación e investigación y difusión del conocimiento que ofrecen los grupos de investigación.

EI PCT recibe las ofertas y demandas de colaboración, ofrece asesoramiento específico, negocia y realiza trámites para implementar acuerdos.

Los entregables del PCT son los siguientes:

- Invenciones generadas en los proyectos PCT.

- Patentes, para el registro de la propiedad industrial.

- Contratos, a través de la cual se intermedia entre la empresa y el investigador.

- Asesoría económica, negocios y gestión para los grupos que participen en los proyectos.

\subsection{Capacidad de desarrollo de patentes}

Un área de patentes de la UNMSM gestiona las solicitudes y orienta y apoya al investigador en la tramitación y registro de sus inventos. La UNMSM debe elaborar un catálogo I+D+i+e con información 
Figura 2. Desarrollo esperado de PCT hasta llegar a Ciudad del Conocimiento

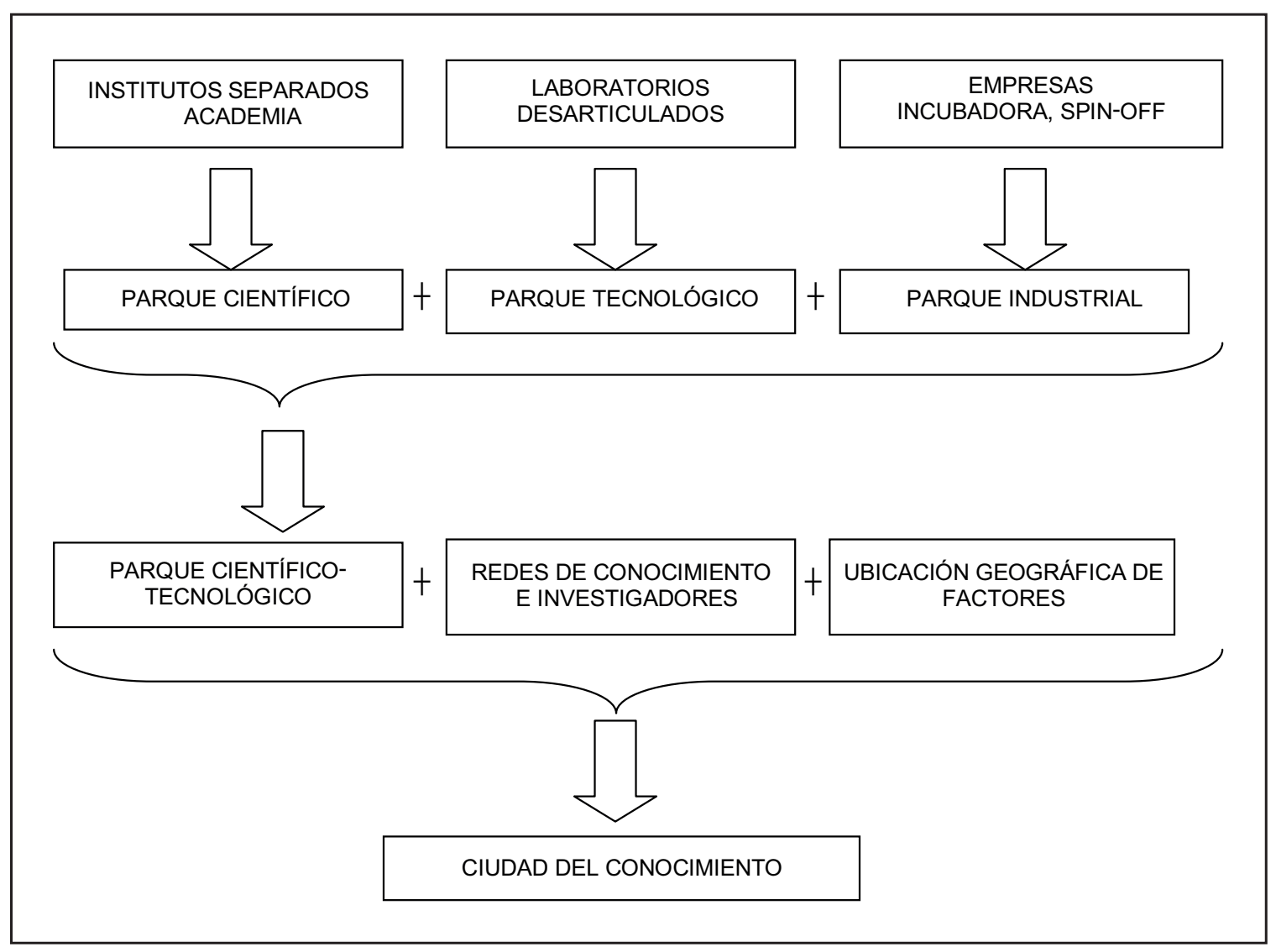

Fuente: Elaboración propia.

de todos los proyectos y grupos de investigación, a fin difundirlo ante el empresariado sobre los servicios, acercando la investigación universitaria a las empresas.

El catálogo I+D+i+e de la universidad representa una herramienta esencial para los grupos de investigación, que han de utilizar su potencial y ponerlo en práctica en la empresa, y para la oficina de transferencias a fin que relaciones las líneas de investigación con los requerimientos tecnológicos. El catálogo significa la oferta de la Universidad y se debe manejar con criterio de eficiencia económica, además se mantiene objetivos de mejora continua y adaptación al ámbito empresarial, con compromiso en el diseño de procesos y servicios para satisfacer las necesidades de los clientes empresariales.

\subsection{Capacidad de crear relaciones con asociaciones en innovación}

A través de la Asociación International de Ciencia y Tecnología (International Association of Science and Technology Parks IASP), se conecta a profesionales a cargo de parques científico-tecnológicos de todo el mundo, proporcionando servicios para el impulso, crecimiento y eficacia de sus miembros.

Los objetivos de la IASP son:

- Contribuir al desarrollo de nuevos parques científico y tecnológicos.

- Fomentar las asociaciones entre las fuerzas motrices en la ciencia y parques tecnológicos e incubadoras.

- Fomentar la creación de redes internacional entre sus miembros.

\section{EN EL CAMINO DE LA EVOLUCIÓN DEL PCT}

Uno de los caminos que puede seguir, es pasar de un modelo de PCT evolucionando hacia estados superiores [7], como la ciudad del conocimiento [2], en una secuencia semejante a la mostrada en la Figura 2.

Se define a la Ciudad del Conocimiento como un territorio geográfico donde se asientan academia y empresas con el fin de desarrollar una economía 
basada en el conocimiento, de manera planeada, con el apoyo de gobierno.

Las características de la Ciudad del Conocimiento son las siguientes:

- Los actores son académicos investigadores y emprendedores.

- Posee ventajas competitivas para atraer a actores de otras localidades [19].

- Posee recursos competitivos para establecer capacidades formativas y redes de conocimiento.

- Gestión y funcionamiento eficaz para el adecuado flujo de conocimiento entre los actores.

- El espacio geográfico, recursos y factores son la base de la generación de conocimiento.

- Enlaza redes de investigadores con las organizaciones y la infraestructura para el desarrollo de las innovaciones.

\section{CONCLUSIONES}

1. Los lineamientos de un Proyecto de Parque Científico y Tecnológico sugieren un elevado potencial de aporte a la consolidación de la Universidad como organización creadora de conocimiento, que realiza actividades de investigación y apoya el surgimiento y desarrollo de negocios, con la tecnología adecuada para el desarrollo de la economía del país.

2. Respecto a la formulación del Proyecto PCT dirigido por la Facultad de Ingeniería Industrial de San Marcos, se estima que el PCT es un medio para el desarrollo nacional, cuyo estudio, diseño, implantación y funcionamiento, ha de ser asumido como parte del proceso para llegar a niveles de sectores y clústeres industriales competitivos y ciudades del conocimiento.

3. Sobre la gestión y organización, existen diferentes modelos que pueden aplicarse para la gestión de PCT. En el caso de FII-UNMSM, el modelo adecuado comprendería la relación universidad-gobierno-empresa, de manera que cada uno aporte las tareas y actividades que permitan la creación de sinergias compartidas.

4. Para potenciar las características competitivas que permitan el planteo de convenios con empresas e instituciones, se considera que el PCT busca sentar las bases de conocimiento científico aplicado, para que se desarrollen y consoliden empresas competitivas, cuyos resultados han de incrementar la producción, crear empleo productivo, generar exportaciones no tradicionales y de valor agregado tecnológico.

5. Un PCT tiene el efecto complementario de generar conocimiento técnico y científico y de estimular el flujo del saber entre academia, empresa y gobierno, lo que se traduce en capacidad de generar nuevos emprendimientos y nuevos tipos de negocio que permitan mantener el ritmo de crecimiento de los sectores industriales del país.

\section{REFERENCIAS BIBLIOGRÁFICAS}

[1] Afuah, Allan (1997). La dinámica de la innovación organizacional. Oxford University Press. México.

[2] Aguirre, Juan (2004). "Monterrey: Ciudad Internacional del Conocimiento", en Foro Consultivo Científico y Tecnológico, Comisión de Economía, Cámara de Diputado. México D. F.

[3] Banco Mundial (2008). "International Good Practice For Establishment Of Sustainable It Parks. Review of Experiences in Select Countries, Including Three Country Case Studies: Vietnam, Russia \& Jordan". Extraído el 14-04-12 desde: http://www. infodev.org/en/ Document.557.pdf

[4] Bhidé, Amar (2000). Origen y evolución de nuevas empresas. Oxford University Press. México.

[5] Casallas, Plata, Pineda (2011). "Parques Tecnológicos como Mecanismo de Integración entre Universidades, Empresas y el Estado: retos para Colombia". VI Congreso Internacional de la Red de Investigación y Docencia en Innovación Tecnológica - RIDIT Innovación, Empresa y Región Manizales. Colombia.

[6] Casallas, Plata, Arias (2010). Parques Tecnológicos, una plataforma de tecnología e innovación: conceptualización y experiencias internacionales.. Memorias de la Feria de Trabajos de Investigación en IEEE Workshop 2010, Universidad Piloto de Colombia.

[7] Castells, Manuel (1989). La ciudad informacional: tecnologías de la información, reestructuración económica y el proceso urbano regional. Alianza Editorial. Madrid, España.

[8] Domínguez, Álvarez, García, Domínguez, Ruiz (1998). Dirección de Operaciones. Aspectos estratégicos en la producción y los servicios. Ed. McGraw-Hill. España. 
[9] DPN (2011) "Plan Nacional de Desarrollo: Prosperidad para Todos (2010-2014) - Capítulo III-Crecimiento sostenible y competitividad. Pp. 48-248. Extraído el 14-08-12 desde: http://www. dnp.gov.co/portalweb/linkclick.

[10] Etzkowitz, y Leydesdorff (2000). "The dynamics of innovation: from National Systems and "Mode 2" to a Triple Helix of university-industrygovernment relations". Extraído el 12-11-12 desde: de http://cmapspublic3.ihmc.us/.

[11] Espinoza, Nemesio (2008). Parques Tecnológicos en el Perú. Extraído el 19-11-12 desde: http://nespinozah.blogspot.com/2008/04/ parques-tecnolgicos-en-el-peru.html

[12] International Association of Science and Technology Parks IASP (2002). "About Science and Technology Parks-Definitions". Extraído el 8-9-12 desde: http://www.iasp.ws/publico/

[13] Kista Science City. (2010). "Kista Science City". Extraído el 19-5-12 desde: http://www.kista. com/adimo4/site/kista/web/default.

[14] Leydesdorff (2006). "The Knowledge-Based Economy and the Triple Helix Model". Extraído el 10-7-12 desde: http://www.leydesdorff.net/ arist09/arist09.pdf.

[15] Morales (2000) "Libre Empresa" en Política de Parques Tecnológicos. Vol. 8 N. ${ }^{\circ} 1$, Enero - Junio de 2011 Ministerio de Desarrollo Económico. Colombia.

[16] National Research Council. (2009). "Understanding Research and Technology Parks.Global Best Practice, Report of Symposium National Academies Press".
Extraído el 11-10-12 desde: http://www.nap. edu/catalog.php.

[17] Parcbit. Parque de Innovación Tecnológico Balear. Extraído el 8-8-12 desde: http://www. parcbit.es/wparcbitfront/Index.jsp

[18] Porter, Michael (1996). Ser Competitivo. Ediciones DEUSTO, España.

[19] Porter, Michael (1996). La ventaja competitiva de las naciones. Extraído el 26-9-12 desde: http://www.micheltissot.com/academia/ competyprod/Modulo\%204.2.pdf

[20] Roussell, Saad, Erickson (1991). Tercera generación de I+D. Su integración en la estrategia de negocio. Ed. McGraw-Hill. España.

[21] Unesco (2008). "Science and Technological Parks: Concept and Definition". Extraído el 147-12 desde: http://www.unesco.org/new/en/ natural-sciences/science-technology/sciencepolicy/thm_innov/unispar/sc_parks/concept. shtml

[22] UNMSM -Vicerectorado de Investigación (2013). "Plan Estratégico VRI 20122016". Extraído el 29-8-13 desde: http:// vrinvestigacion.unmsm.edu.pe/publicaciones/ libros/319-plan-estrategico-vri-2012-2016.

[23] Spolidoro y Audy (2008) .Parque Científico e Tecnológico da PUCRS. Extraído el 22-5-12 desde: http://www.pucrs.br/edipucrs/tecnopuc/ frame.htm

[24] Tecnoparque SENA (2009). "Tecnoparque Colombia". Extraído el 7-8-12 desde: http:// www.tecnoparquecolombia.edu.co/ 